\title{
Qualidade de vida de idosos brasileiros e portugueses: uma análise comparativa
}

Quality of Life in Older Adults in Brazil and Portugal: A Comparative Analysis

Análisis comparativo de la calidad de vida de los adultos mayores de Brasil y Portugal

Como citar este artigo: Sousa, Fabianne de Jesus Dias de; Oliveira, Catarina Resende de; Pinto, Anabela Mota; Rodrigues, Victor; Gonçalves, Lucia Hisako Takase; Gamba, Mônica Antar. Qualidade de vida de idosos brasileiros e portugueses: uma análise comparativa. Revista Cuidarte. 2021;12(1):e1230.

http://dx.doi.org/10.15649/cuidarte.1230

Revista Cuidarte

doil Rev Cuid. Ene. - Abril. 2021; 12(1): e1230

http://dx.doi.org/10.15649/cuidarte.1230

E-ISSN: 2346-3414

(1) Fabianne de Jesus Dias de Sousa

(1) Catarina Resende de Oliveira ${ }^{2}$

(1) Anabela Mota Pinto ${ }^{3}$

(1) Victor Rodrigues ${ }^{4}$

(1) Lucia Hisako Takase Gonçalves ${ }^{5}$

(1) Mônica Antar Gamba ${ }^{6}$

1 Universidade Federal do Pará, Belém, Brasil.

E-mail:fabiannesousa@hotmail.com Autor para correspondência

2 Universidade de Coimbra, Coimbra, Portugal. E-mail:

catarina.n.oliveira@gmail.com

3 Universidade de Coimbra, Coimbra, Portugal. E-mail: apinto@uc.pt

4 Universidade de Coimbra, Coimbra, Portugal.

E-mail:Vrodrigues@netcabo.pt

5 Universidade Federal do Pará, Belém, Brasil. E-mail: Ihtakase@gmail.com

6 Universidade Federal de São Paulo, São Paulo, Brasil.

E-mail: antar.gamba@unifesp.br

\section{Resumo}

Introdução: A longevidade tornou-se uma das maiores conquistas da segunda metade do século XX. Objetivo: Comparar a qualidade de vida de idosos brasileiros e portugueses usuários da atenção primária à saúde. Materiais e Métodos: Estudo quantitativo, transversal e comparativo, aplicado a amostra probabilística englobando 294 idosos acompanhados pela Saúde da Família divididos em dois grupos: 130 idosos em Benevides/Brasil, e 164 em Coimbra/Portugal, no período de 2015-2017. A caracterização sociodemográfica e avaliação da qualidade de vida foram obtidos pelo instrumento World Health Organization Questionnaire of Quality of Life (WHOQOL-bref), consta de 26 questões divididas em quatro domínios: físico, psicológico, relações sociais e meio ambiente. Aplicado o Teste $U$ de Mann-Whitney para análise estatística comparativa. Resultados: Nos grupos verificou-se predomínio do sexo feminino, casados, aposentados e com baixo grau de escolaridade (1 a 4 anos). A média da distribuição etária foi 70 anos para brasileiros e 76 anos para portugueses; na comparação da qualidade de vida, os idosos brasileiros obtiveram os melhores escores no domínio psicológico $(79,1)$ enquanto portugueses tiveram melhor escore no domínio meio ambiente $(65,6)$. Em Benevides o escore mais baixo foi o domínio meio ambiente $(56,3)$ e, em Coimbra, o domínio com escore mais baixo foi o físico $(60,7)$. Discussão: A qualidade de vida no envelhecimento é importante preditor para a preservação da autonomia do idoso. Conclusões: Estes resultados ampliam a concepção da importância da atenção integral no processo de envelhecimento com vista a proporcionar melhor qualidade de vida contribuindo para satisfazer as necessidades especificas da população idosa.

Palavras-chave: Qualidade de Vida; Saúde do Idoso; Envelhecimento Saudável; Comparação Transcultural.

Recibido: 28 de abril de 2020 Aceptado: 16 de septiembre de 2020 Publicado: 25 de febrero de 2021 $\square *$ Correspondência

Fabianne de Jesus Dias de Sousa E-mail:fabiannesousa@hotmail.com 


\section{Quality of Life in Older Adults in Brazil and Portugal: A Comparative Analysis}

\section{Abstract}

Introduction: Longevity is one of the greatest achievements in the last half of the $20^{\text {th }}$ century. Objective: To compare the quality of life in older adults in Brazil and Portugal receiving primary health care. Materials and Methods: A comparative cross-sectional quantitative study was conducted with a probability sample of 294 older adults, who had been assisted with the Family Health strategy, being divided into two groups: 130 older adults living in Benevides, Brazil and 164 in Coimbra, Portugal during 2015 and 2017. The sociodemographic characterization and the quality of life assessment were determined through the WHO Quality of Life-BREF (WHOQOL-BREF), which consists of 26 questions assessing four domains: physical health, psychological health, social relationships and environment. The Mann-Whitney $U$ test was applied for comparative statistical analysis. Results: There was a prevalence of married, retired women with a lower level of education (1 to 4 years) in the groups. The average age was 70 years in Brazil and 76 years in Portugal. In terms of quality of life, Brazilian older adults got better scores in the psychological health domain (79.1\%) while Portuguese older adults scored best in the environmental domain (65.6\%). The lowest scores were found in the environmental domain in Benevides (56.3\%) and in the physical health domain in Coimbra (60.7\%). Discussion: Quality of life in aging is a major predictor for the preservation of autonomy in older adults. Conclusion: These results help to broaden the importance of comprehensive care in the aging process to provide a better quality of life, which contributes to meeting the specific needs of older population.

Keywords: Quality of Life; Health of the Elderly; Healthy Aging; Cross-cultural Comparison.

\section{Análisis comparativo de la calidad de vida de los adultos mayores de Brasil y Portugal}

\section{Resumo}

Introducción: La longevidad se ha convertido en uno los mayores logros de la segunda mitad del siglo XX. Objetivo: Comparar la calidad de vida de los adultos mayores de Brasil y Portugal que reciben atención primaria de salud. Materiales y métodos: Se realizó un estudio cuantitativo transversal comparativo con una muestra probabilística de 294 adultos mayores que fueron acompañados por la estrategia de Salud Familiar y divididos en dos grupos: 130 adultos mayores ubicados en Benevides, Brasil y 164 en Coímbra, Portugal durante 2015 y 2017. La caracterización sociodemográfica y la evaluación de la calidad de vida se obtuvieron mediante el Cuestionario de Calidad de Vida de la Organización Mundial de la Salud (WHOQOL-BREF), que consta de 26 preguntas divididas en cuatro áreas: salud física, salud psicológica, relaciones sociales y ambiente. Se aplicó la prueba U de Mann-Whitney para el análisis estadístico comparativo. Resultados: En los grupos se observó el predominio de las mujeres casadas, jubiladas y con baja escolaridad (entre 1 y 4 años). La edad media fue de 70 años en Brasil y 76 años en Portugal. En cuanto a la calidad de vida, los adultos mayores brasileños obtuvieron mejores puntuaciones en el área de salud psicológica (79.1\%) mientras que los adultos mayores portugueses tuvieron la mejor puntuación en el área ambiental (65.6\%). Las puntuaciones más bajas se presentaron en el área ambiental en Benevides (56.3\%) y en la salud física (60.7\%) en Coímbra. Discusión: La calidad de vida en el proceso de envejecimiento es un importante predictor para la preservación de la autonomía de los adultos mayores. Conclusiones: Estos resultados amplían la concepción de la importancia de la atención integral en el proceso de envejecimiento para brindar una mejor calidad de vida, lo que contribuye a satisfacer las necesidades específicas de la población de edad avanzada.

Palabras clave: Calidad de Vida; Salud del Anciano; Envejecimiento Saludable; Comparación Transcultural. 


\section{Introdução}

O aumento da esperança de vida em todo o mundo tem suscitado debates na comunidade científica, desafiando a prerrogativa de viver mais tempo, porém com qualidade de vida, apontando formas de promover um envelhecimento mais ativo e saudável. A Organização Mundial de Saúde (OMS) define qualidade de vida pela maneira como o indivíduo percebe sua posição na vida, no contexto da cultura e sistemas de valores nos quais vive e em relação aos seus objetivos, expectativas, padrões e preocupações'.

O desafio do século XXI será melhorar a prestação de cuidados de vida e saúde da crescente população idosa. Na América Latina e Caribe, o número de pessoas muito idosas ( $\geq 85$ anos) passou de 6,5\% da população geral em 1950 para 11,1\% em 2000, com projeção de 18,1\% para $2050^{2}$.

No Brasil, em 2010 os idosos representavam 8,6\% da população geral, podendo em 2020 atingir $20 \%$. Prevê-se que em 2050 que a população jovem com idade entre 0 e 14 anos representem $13,1 \%$ da população total, enquanto o estrato idoso ( $\geq 60$ anos) atingirá $22,7 \%$, justificando a inversão da pirâmide populacional ${ }^{3,4,5}$.

Em Portugal, no ano de 2010, os idosos ( $\geq 65$ anos) representavam $19 \%$ da população geral, com esperança média de vida de 79,8 anos. O índice de envelhecimento passou de $27,5 \% \mathrm{em}$ 1961 para 144\% em 2015. O fenômeno de envelhecimento é mais acentuado nas mulheres, o que se repete no grupo dos idosos mais idosos (idade $\geq 85$ anos), com uma taxa de $12,0 \%$ da população geral, índice de países desenvolvidos ${ }^{6}$.

A atenção primária em saúde é a ordenadora de redes de atenção em países desenvolvidos e em desenvolvimento. Na presente análise, o Brasil, liderado pelo Ministério da Saúde, considera o trabalho em equipe multidisciplinar como elemento central na atenção primária. Portugal, por sua vez, além de contar com o trabalho multidisciplinar, é um dos países que mais desenvolvem instrumentos de avaliação para serem adotados nos Cuidados de Saúde Primários (CSP)․

Em ambos os países, os sistemas de saúde se guiam por ideais comuns, pois ambos defendem estratégias que fortaleçam a atenção primária. Estudos revelam que Brasil e Portugal discutem práticas que facilitam o desempenho dos profissionais com vistas a melhorar a coordenação dos serviços de saúde ${ }^{8-10}$, sendo também relevante estudar a qualidade de vida dos idosos desses países para encontrar linhas orientadoras para o desenvolvimento de programas e ações que beneficiem a população idosa, com políticas públicas e programas promotores de envelhecimento ativo e saudável.

Nesse contexto, a avaliação da qualidade de vida da pessoa idosa abrange alguns aspectos, tais como: capacidade funcional; nível socioeconômico; estado psicológico; interação social; atividade intelectual; autocuidado; apoio familiar; valores socioculturais, éticos e religiosos; processo de morte e morrer; estilo de vida; satisfação com o emprego e/ou atividades de vida diárias e, o ambiente em que se vive/frequenta. Sabe-se que, as alterações ocasionadas pelo processo fisiológico e patológico do envelhecimento corroboram para uma queda na qualidade de vida do idoso, justificando a relevância de estudos que suportem estratégias e ações voltadas à prevenção e manutenção de um processo de envelhecimento 
ativo e saudável ${ }^{11}$.

Neste contexto, vários instrumentos são utilizados com o intuito de avaliar a qualidade de vida, entre eles, o World Health Organization Questionnaire of Quality of Life (WHOQOL-Bref), forma abreviada do WHOQOL-100, composto por 26 questões, sendo duas gerais, relacionadas à qualidade de vida e à saúde global e, 24 que compõem os quatros domínios: Físico; Psicológico; Relações Sociais e Meio Ambiente ${ }^{12}$.

Assim, o presente estudo teve como objetivo comparar a qualidade de vida de idosos brasileiros e portugueses usuários da atenção primária à saúde.

\section{Materiais e métodos}

Estudo com abordagem quantitativa, transversal e comparativo ${ }^{13}$ aplicado a amostra probabilística, e desenvolvido com idosos atendidos em unidades de atenção primária de saúde de dois países, Brasil e Portugal: respectivamente um na unidade de saúde da família do Município de Benevides, Pará, Brasil, e outro na unidade de saúde familiar do Concelho de Coimbra, Portugal. O estudo foi realizado em dois momentos: inicialmente em Benevides (Pará, Brasil) no período de julho de 2015 a fevereiro de 2016; e em Coimbra (Portugal), no período de abril a maio de 2017.

A população geral de Benevides é de 51.651 habitantes, com população idosa de 4.856 indivíduos cadastrados na unidade de saúde em estudo ${ }^{14}$. Em Coimbra, da população de 105.842 habitantes, o estrato de idosos é de 6.825 indivíduos cadastrados na unidade em estudo ${ }^{15}$.

A pesquisa foi realizada com 294 idosos (130 idosos brasileiros e 164 idosos portugueses) de ambos os sexos, sendo 55 homens e 75 mulheres brasileiros e 75 homens e 89 mulheres portugueses, após realizado o sorteio. A amostra foi calculada pela técnica de amostragem aleatória estratificada por unidade de saúde e sexo conforme expressão ${ }^{16}$ :

$\mathrm{n}=\frac{\mathrm{N} \times \mathrm{n}_{0}}{\mathrm{~N}+\mathrm{n}_{0}}$ em que $n_{0}=\frac{1}{e^{2}}=\frac{1}{0,05^{2}}$

é o erro amostral e, Né o número total de idosos cadastrados e acompanhados, com e sendo o erro amostral; considerou-se nesse caso, nos idosos brasileiros, erro amostral de 0,64, e portugueses erro amostral de 0,78; em ambos os países 1,732; p=0,085.

Adotou-se como critério de inclusão dos idosos para ambos os países: ser residente e/ou atendido na área adstrita às unidades de saúde selecionadas para o estudo; no caso de idosos brasileiros, ter idade igual ou superior a 60 anos, enquanto em Portugal ter idade igual ou superior a 65 anos, segundo o critério da OMS'. O critério de exclusão: foi não encontrar os idosos no seu domicílio.

Para avaliar a qualidade de vida aplicou-se o World Health Organization Questionnaire of Quality of Life (WHOQOL-bref) em sua versão abreviada e validada no idioma português de cada país, além da aplicação de um instrumento contendo dados sociodemográficos (sexo, faixa etária, estado conjugal, escolaridade e ocupação).

OWHOQOL-bref é um instrumento composto de 26 questões: duas gerais acerca de percepção de qualidade de vida e de estado de saúde; e 24 questões distribuídas em quatro domínios: físico, psicológico, relações sociais e meio ambiente, como se discrimina a seguir: a) Domínio físico (1), com sete questões, avalia dor e desconforto, energia/fadiga, sono/repouso, mobilidade, 
atividades da vida cotidiana, dependência de medicação ou de tratamentos e capacidade de trabalho. b) Domínio psicológico (2), com seis questões, focaliza sentimentos positivos e negativos, como: pensar, aprender, memória/concentração, autoestima, imagem corporal e aparência; espiritualidade, religião/crenças pessoais. c) Domínio relações sociais (3), com três questões, aborda as relações pessoais, suporte (apoio) social, atividade sexual. d) Domínio meio ambiente (4), com oito questões, inclui aspectos como segurança física/proteção, ambiente no lar, recursos financeiros; cuidados de saúde e sociais: disponibilidade/qualidade, oportunidade de adquirir novas informações e habilidades, participação em oportunidades de recreação/lazer; ambiente físico: poluição, ruído, trânsito, clima e transporte. No WHOQOL-bref, a qualidade de vida é avaliada por meio da escala Likert, cujas respostas às questões em escores variam de 1 a 5, assinalando para condições piores o escore 1, e para as melhores o escore 5. 0 resultado de uma avaliação por esse instrumento é convertido em escala centesimal de 0 a 100, sendo o maior valor correspondente a melhor percepção da qualidade de vida ${ }^{17}$.

As características psicométricas do instrumento validado no Brasil preenchem os critérios de validade discriminante, concorrente e de conteúdo, e de confiabilidade por consistência interna de valor Alfa de Cronbach de $a>0,80^{17}$.A versão validada em português de Portugal, utilizada com idosos de Coimbra, também teve sua validade conferida semelhantemente ao procedimento adotado na versão brasileira, incluindo a confiabilidade por consistência interna de valor Alfa de Cronbach de $a>0,92^{18}$.

A escolha pelo WHOQOL-bref ${ }^{19}$ se deu pelas boas características psicométricas e também pela extensão do instrumento com 26 questões, adequado para ser usado com idosos sem exaurilos. O instrumento foi aplicado com assessoramento da pesquisadora por haver na amostra pessoas não alfabetizadas ou semianalfabetas.

Iniciava-se a coleta de dados com a leitura do Termo de Consentimento Livre e Esclarecido, prestando os esclarecimentos necessários; após a assinatura do termo, passava-se a coletar os dados de identificação e de qualidade de vida. A entrevista se dava nos consultórios de enfermagem das unidades de saúde, ou nos domicílios, conciliando o interesse dos idosos com a conveniência para a pesquisadora.

Os dados coletados foram transcritos com dupla digitação independente utilizando o programa Microsoft Office Excel 2010, e depois analisados por meio do software Statistical Package for the Social Sciences (SPSS) versão 20.0 para Windows. Na avaliação dos escores dos domínios de qualidade de vida segundo o WHOQOL-bref, para comparar as variáveis entre os grupos foi utilizado o Teste $U$ de Mann-Whitney (constatada ausência de normalidade dos dados em $\mathrm{T}=1,08)$; por estarmos comparando os escores de qualidade de vida entre grupos de idosos de Coimbra e Benevides, foi adotado o nível de significância de $p \leq 0,05$.

O estudo foi submetido ao Comitê de Ética em Pesquisa da Universidade Federal de São Paulo, que o aprovou no Parecer $n^{\circ}$ 990.544, CAAE: 41557915.8.0000.5505). Igualmente submetido ao Comitê de Ética em Saúde da Agência Regional em Saúde do Centro (ARSC) de Portugal, foi aprovado no Parecer $n^{\circ} 34 / 2017$. Foram garantidos aos participantes os princípios éticos de respeito à autonomia, beneficência, não maleficência e confidencialidade. Os participantes assinaram o Termo de Consentimento Livre e Esclarecido (TCLE) em duas vias. No caso dos idosos não alfabetizados foi utilizada a impressão digital no lugar da assinatura. 


\section{Resultados}

Quanto à caracterização dos idosos amostrados nos grupos, houve predomínio do sexo feminino, de casados, aposentados e com baixo grau de escolaridade. Em termos de distribuição etária, prevaleceu a faixa etária de 60 a 64 anos entre brasileiros, enquanto entre os portugueses a maioria estava na faixa acima de 80 anos (Tabela 1).
Em termos de distribuição etária, prevaleceu a faixa etária de 60 a 64 anos entre brasileiros, enquanto entre os portugueses a maioria estava na faixa acima de 80 anos

Tabela 1. Perfil dos idosos quanto a sexo, faixa etária, estado civil, escolaridade e ocupação nos contextos de Coimbra-Portugal, e de Benevides - PA, Brasil, 2016-2017

\begin{tabular}{|c|c|c|c|c|c|}
\hline \multirow[t]{2}{*}{ Variáveis } & \multicolumn{2}{|c|}{ Coimbra (Portugal) } & \multicolumn{2}{|c|}{ Benevides (Brasil) } & \multirow[t]{2}{*}{$\begin{array}{l}\text { Valor } \\
\text { de } \mathrm{p}^{*}\end{array}$} \\
\hline & n (164) & $\%$ & n (130) & $\%$ & \\
\hline Sexo & & & & & 0,32 \\
\hline Masculino & 75 & 45,8 & 55 & 42,3 & \\
\hline Feminino & 89 & 54,2 & 75 & 57,7 & \\
\hline Faixa Etária & & & & & 0,08 \\
\hline $60 \nmid 64$ anos & - & - & 50 & 38.4 & \\
\hline $65-69$ anos & 45 & 29,2 & 23 & 17,6 & \\
\hline $70 \dashv 79$ anos & 38 & 24,7 & 37 & 28,0 & \\
\hline$\geq 80$ anos & 81 & 46,1 & 21 & 16,0 & \\
\hline Média \pm Desvio Padrão & \multicolumn{2}{|c|}{$76,7 \pm 7,9$ anos } & \multicolumn{2}{|c|}{$70,5 \pm 8,9$ anos } & \\
\hline Estado conjugal & & & & & $<0,01$ \\
\hline Casado & 97 & 59,0 & 25 & 31,0 & \\
\hline Viúvo & 60 & 36,5 & 33 & 41,0 & \\
\hline Divorciado/Separado & - & - & 10 & 12,0 & \\
\hline Solteiro & 07 & 4,5 & 13 & 16,0 & \\
\hline Escolaridade & & & & & $<0,03$ \\
\hline Nenhuma & 10 & 6,4 & 23 & 28,0 & \\
\hline Primário incompleto (1 a 4 anos) & 103 & 66,9 & 43 & 53,0 & \\
\hline Primário completo (5 a 6 anos) & 10 & 6,4 & 09 & 11,0 & \\
\hline Ginásio completo (7 a 9 anos) & 07 & 4,5 & 01 & 2,0 & \\
\hline Ensino médio (10 a 12 anos) & 16 & 10,4 & 03 & 4,0 & \\
\hline En sino superior/Pós -graduação & 18 & 11,8 & 03 & 4,0 & \\
\hline Ocupação & & & & & $<0,47$ \\
\hline Aposentado & 116 & 70,7 & 66 & 81,0 & \\
\hline Pensionista & 0 & 0 & 11 & 14,0 & \\
\hline Dolar /Doméstica & 30 & 19,5 & 01 & 1,0 & \\
\hline Trabalhador de serviços/administração pública & 08 & 6,2 & 02 & 2,0 & \\
\hline Trabalhador nível superior & 10 & 6,2 & 01 & 1,0 & \\
\hline
\end{tabular}

*Teste Qui-quadrado

Fonte: Elaboração dos autores 
Os resultados da comparação entre grupos de idosos e seus respectivos escores médios nos diferentes domínios da qualidade de vida demonstraram significância estatística $(p \leq 0,005)$. O grupo de Coimbra teve melhor escore no domínio 4 (Meio ambiente) $(65,6 \pm 12,5 ; p<0,001)$, enquanto Benevides teve os melhores escores nos domínios: 2 (Psicológico) $(79,1 \pm 16,7 ; p<0,001)$ e 3 (Relações sociais) (75 $\pm 16,7 ;$ p $<0,001)$. Em Coimbra, o domínio com escore médio mais baixo foi o domínio 1 (Físico) $(60,7 \pm 21,4 ;$ p $<0,116)$, enquanto em Benevides foi o domínio 4 (Ambiente) $(56,3 \pm 18,7 ; p<0,001)$ (Figura 1).

A
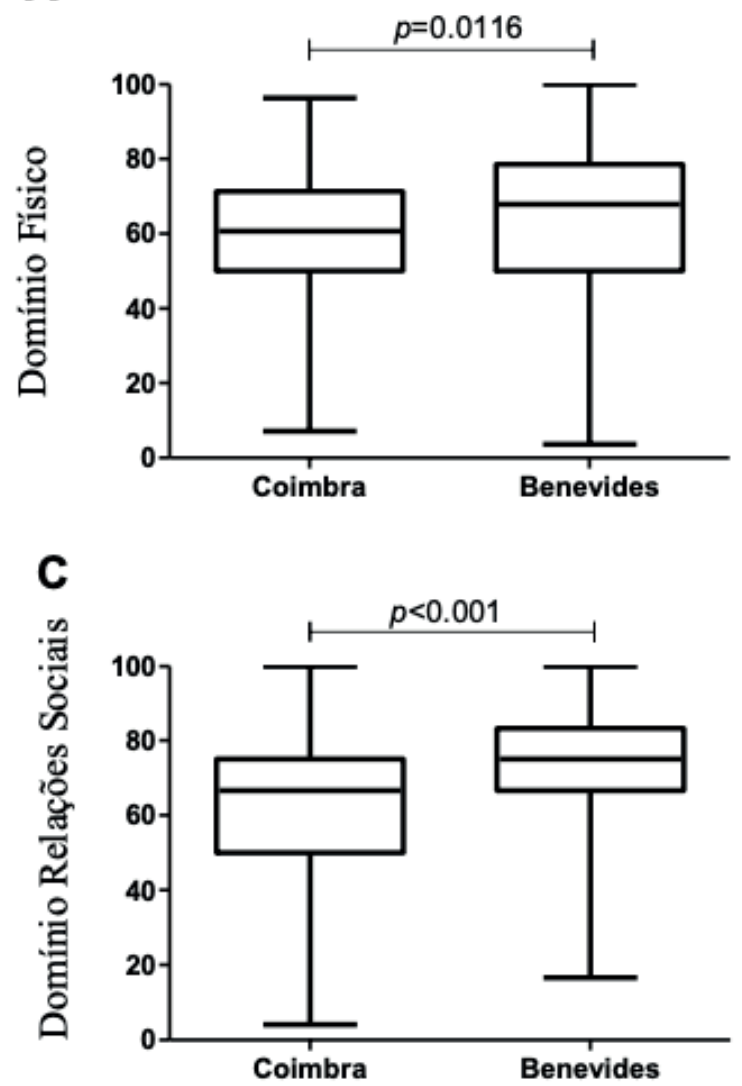

B

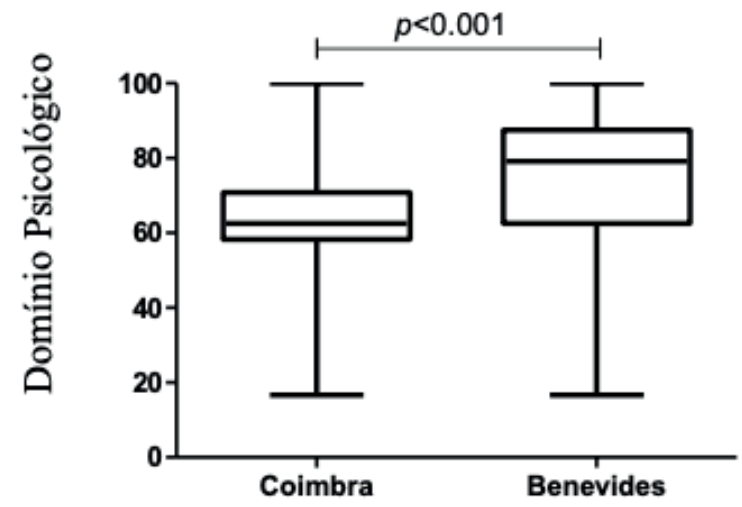

D

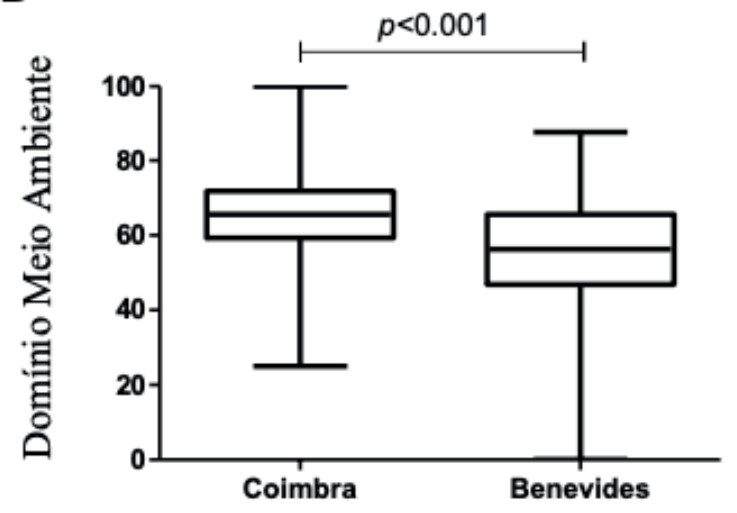

Figura 1. Box Plot comparativo da avaliação da Qualidade de vida de idosos segundo os domínios do WHOQOL-bref. Coimbra, Portugal e de Benevides-PA, Brasil, 2016 - 2017 *Teste de Mann-Whitney Fonte: Elaboração dos autores

A descrição da qualidade de vida dos idosos, mensurada pelo WHOQOL-Bref, evidenciou entre os domínios valores de mediana superiores a 56,3 (Tabela 2). 
Tabela 2. Descrição da Qualidade de vida de idosos segundo os domínios do WHOQOLBref. Coimbra, Portugal e de Benevides-PA, Brasil, 2016 - 2017

\begin{tabular}{lccccc}
\hline $\begin{array}{l}\text { Concelho } \\
\text { /Município }\end{array}$ & Domínios & Freq. & Mediana & $\begin{array}{c}\text { Desvio- } \\
\text { Quartílico }\end{array}$ & Valor de p \\
\hline Coimbra & Geral $^{* *}$ & 164 & 57,1 & 12,6 & $<0,0004$ \\
Benevides & Geral* $^{*}$ & 130 & 67,6 & 18,8 & $<0,0001$ \\
Coimbra & Físico & 164 & 60,7 & 21,4 & $<0,0001$ \\
Benevides & Físico & 130 & 67,9 & 28,6 & $<0,0001$ \\
Coimbra & Psicológico & 164 & 62,5 & 12,5 & $<0,0001$ \\
Benevides & Psic ológico & 130 & 79,1 & 16,7 & $<0,0001$ \\
Coimbra & Relações Sociais & 164 & 66,6 & 25,0 & $<0,0001$ \\
Benevides & Relações Sociais & 130 & 75,0 & 16,7 & $<0,0001$ \\
Coimbra & Meio Ambiente & 164 & 65,6 & 12,5 & $<0,0001$ \\
Benevides & Meio Ambiente & 130 & 56,3 & 18,7 & $<0,0001$ \\
\hline
\end{tabular}

*Teste de U Mann-Whitney

**Domínio Geral é composto: Questão 1(Q1) doWHOQOL-Bref:Como vocêavaliaria sua qualidade de vida? e, Questão 2 (Q2) do WHOQOL-Bref: Quão satisfeito você está com a sua saúde?

Fonte: Elaboração dos autores

\section{Discussão}

A qualidade de vida é um dos fatores diretamente ligados ao processo de envelhecimento da população ${ }^{20}$, devendo ser considerada em programas e políticas sociais e de saúde com vistas à integralidade do cuidado prestado à população idosa ${ }^{21}$.

Entre os idosos que participaram no estudo predominou o sexo feminino em ambos os países, corroborando dados da literatura mundial que revelam a feminização da velhice, tendo a mulher maior sobrevida e cuidar mais de sua saúde. Além de fatores biológicos, é possível que a longevidade maior da mulher se deva aos programas de saúde mais voltados para esse segmento, facilitando-lhe acesso aos serviços de saúde ${ }^{22,23}$.

No tocante à estrutura etária dos idosos brasileiros, são mais jovens, grande parte encontrase na faixa dos 60 a 69 anos, enquanto os idosos portugueses, mais velhos, na faixa etária octogenária, resultados que corroboram achados internacionais de relacionar cuidados de saúde com aumento da longevidade. Essa maior longevidade pode resultar na incapacidade funcional, afetando a autonomia e, consequentemente, sua qualidade de vida. Entretanto, estudos evidenciam que a percepção da qualidade de vida aumenta com o envelhecimento, podendo indicar maior aceitação diante da inexorabilidade do processo ${ }^{23-26}$.

Quanto ao estado conjugal, os resultados do estudo mostraram similaridades entre Brasil e Portugal. Em ambos os países, a viuvez revelou-se importante por aprofundar o isolamento do idoso, devendo ser interpretada com cuidado no domínio referente às relações sociais na qualidade de vida $22,27,28$.

$\mathrm{Na}$ análise da escolaridade observou-se que tanto entre os idosos brasileiros quanto entre os idosos portugueses prevaleceu o índice de baixa escolaridade. Tal resultado corrobora dados 
da literatura mundial, mostrando a baixa escolaridade da população idosa, provavelmente fruto da realidade sociocultural de ambos os países num tempo em que a mulher era educada para ser do lar, enquanto os homens eram responsáveis por trabalhar fora para sustentar a família $25,26,29,30$.

A ocupação revelou similaridade com outros resultados, como maioria de idosos aposentados em ambos os países, o que se equipara aos dados de estudo realizado em Portugal, onde 68,9\% dos idosos vivem de aposentadoria, ou pensão, no caso das viúvas ${ }^{28,31}$, e os de outro estudo realizado no Brasil, onde $88,3 \%$ dos idosos entrevistados eram aposentados ${ }^{26}$.

Comparando os grupos nos quatro domínios da qualidade de vida, os resultados evidenciaram que entre os idosos portugueses o melhor escore incide no domínio Meio ambiente; os investimentos nas áreas da saúde com qualidade, transportes, oportunidades de lazer e recreação, entre outros, são valorizados e disponibilizados à população idosa portuguesa; o pior escore refere- se ao domínio físico. Cabe enfatizar que o avanço da idade pode reduzir a autonomia e a independência funcional desses idosos mais idosos.

Por sua vez, Portugal tem melhor escore no domínio Meio Ambiente. Estudos mostram que os cuidados de saúde primários (CSP) daquele país passam por extensa reforma organizacional, tendo a unidade de saúde familiar (USF) como base da prestação de cuidados de saúde com autonomia assistencial e funcional, trabalhando em rede e próxima do cidadão, destacando-se o trabalho em equipe como elemento central ${ }^{21,29,31}$. Esse resultado assemelha-se ao de estudo realizado com idosos no Concelho de Viana do Castelo ${ }^{31}$, que apresentou melhor escore no domínio Psicológico (revelando a importância das relações sociais e do apoio social), enquanto o pior escore foi no domínio Meio Ambiente, decorrente das condições ambientais.

Os idosos brasileiros tiveram melhores escores nos domínios: Psicológico e Relações Sociais, e pior escore no domínio Meio Ambiente. Desse resultado se infere que os valores maiores nos domínios Psicológico e Relações Sociais significam que a socialização dos idosos pode contribuir nas suas relações sociais, melhorando-Ihes a qualidade de vida. O Meio Ambiente apresentou valor baixo, talvez por várias condições ambientais, inclusive dificuldade de acesso aos serviços de saúde ${ }^{32}$.

Em relação às duas questões gerais do WHOQOL-bref, verificou-se que a maioria dos idosos, tanto brasileiros quanto portugueses, estavam satisfeitos com a sua saúde, apresentando também boa percepção de qualidade de vida geral. Esse resultado corrobora com outros estudos

Verificou-se que a maioria dos idosos, tanto brasileiros quanto portugueses, estavam satisfeitos com a sua saúde, apresentando também boa percepção de qualidade de vida geral. internacionais ${ }^{11,33}$ que evidenciam que grande parte dos idosos possuem uma percepção positiva de sua qualidade de vida.

A qualidade de vida na velhice é hoje um conceito importante no âmbito mundial, uma vez que existe uma nova sensibilidade social para com a velhice, sendo considerada tanto como problema, quanto como desafio para os indivíduos e para a sociedade ${ }^{25}$. Nescse contexto, é relevante esclarecer que, no Brasil, estudo realizado com idosos apontou que as redes de apoio social exercem influência na qualidade de vida dos idosos no tocante a sentimentos positivos ou negativos (domínio psicológico - valores do escore entre 58 e 70 pontos), relações pessoais (domínio relações sociais - valores do escore entre 64 e 71 pontos) e lazer ou recreação (domínio ambiente - valores do escore entre 47 e 62 pontos) ${ }^{29}$. 
O resultado do presente estudo mostrou ser necessário privilegiar ações que melhorem o domínio Meio Ambiente no Brasil, cabendo salientar que, rotineiramente, os idosos brasileiros convivem com medo de violência, falta de assistência médica e de hospitais e escassas atividades de lazer, além de angústia constante, com os baixos valores das aposentadorias e/ou pensões ${ }^{25}$.

No caso de Portugal, o resultado mostrou que o domínio físico deve merecer maior atenção; estudo com idosos naquele país evidenciou que as capacidades de adaptação vão diminuindo à medida que se envelhece ${ }^{26}$. No Brasil, ainda prepondera a assistência fragmentada do cuidado prestado à pessoa idosa, como a consulta motivada basicamente pela necessidade de receber medicamentos, procura de atendimento na agudização da doença e dificuldade no acesso aos serviços de saúde.

Já Portugal presta um cuidado integral ao idoso, com medidas que promovem seu acompanhamento, prevenindo a agudização das doenças. E com base no conhecimento da qualidade de vida da população idosa, os profissionais de saúde podem desenvolver ações que promovam o envelhecimento ativo e saudável.

O processo de envelhecimento inicia-se no nascimento e envolve aspectos individuais e coletivos, influenciando as condições de saúde do idoso ${ }^{21}$. O estudo contribui para que profissionais da saúde, principalmente enfermeiros, reflitam sobre a qualidade de vida, pois promovê-la proporciona maior autonomia à pessoa idosa no seu cotidiano.

Entre as limitações do estudo, destaca-se o fato de ser transversal e realizado apenas numa unidade de saúde em cada um dos países, dificultando a generalização dos achados. Por isso, é de suma importância realizar outros estudos, do tipo longitudinal, com maior ênfase na atenção primária à saúde, com o intuito de apresentar novas contribuições para promover o envelhecimento ativo, melhorando a qualidade de vida de idosos. É necessário ainda realizar novos estudos e aprofundá-los no tocante à qualidade de vida, pois o domínio Ambiente mostrou resultado relevante na comparação entre os dois países economicamente tão diferentes na geopolítica mundial: um país desenvolvido, como Portugal, e outro em desenvolvimento, como o Brasil.

\section{Conclusões}

O presente estudo comparou a qualidade de vida dos idosos brasileiros e portugueses utilizando o instrumento WHOQOL-bref. Enquanto no Brasil a população idosa é majoritariamente de idosos mais jovens, em Portugal predomina o idoso octogenário, apesar de haver similaridades entre os países no aumento da viuvez e feminização da velhice. A comparação entre os domínios de qualidade de vida mostrou que os idosos brasileiros apresentam melhor escore no domínio Psicológico, enquanto os idosos portugueses lograram melhor desempenho no domínio Meio Ambiente.

Considerando que os demais domínios tiveram escores inferiores, a presente avaliação reúne subsídios essenciais para readequar programas de atenção primária de saúde levando em conta as especificidades de cada região.

Em Portugal, o fortalecimento e o incentivo aos serviços de saúde promoveram maior acesso dos idosos aos serviços de saúde e melhor qualidade do serviço prestado pelos profissionais de saúde na atenção primária. No Brasil, apesar de estar em andamento a reestruturação da 
atenção primária com base em novo modelo para monitorar os serviços - o "e-saúde" - salientase a necessidade de fortalecer a atenção primária como elemento central nessa mudança.

Financiamento: Bolsa de doutorado-sanduíche da CAPES - Coordenação de Aperfeiçoamento de Pessoal de Nível Superior. Número do processo: 88881.135327/2016-01.

Conflito de interesses: Os autores declaram que não houve conflitos de interesses.

\section{Referências}

1. TombiniF, CastanhaAC, PadoanER,Lutinski JeSilvaMR.Quality oflife in theelderlywith and without chronic pain. Br J Pain. 2018;1(2):111-5. https://doi.org/10.5935/25950118.20180022

2. Jing $\mathbf{W}$, Willis $\mathbf{R}$, Feng $\mathbf{Z}$. Factors influencing quality of life of elderly people with dementia and care implications: A systematic review. Arch Gerontol Geriatr. 2016;66:23-41. http://dx.doi.org/10.1016/j.archger.2016.04.009

3. Campos ACV, Gonçalves LHT. Aging demographic profile in municipalities in the state of Pará, Brazil. Rev Bras Enferm. 2018;71(Suppl 1):591-8.

http://dx.doi.org/10.1590/0034-71672017-0070

4. Instituto Brasileiro de Geografia e Estatística (IBGE). Projeção da população do Brasil por sexo e idade para o período de 2000/2060: Projeção da população das unidades da federação por sexo e idade para o período de 2000/2030. Rio de Janeiro (RJ): IBGE; 2013.

5. Instituto Brasileiro de Geografia e Estatística (IBGE). Estimativas populacionais para os municípios e para as unidades da federação. Rio de Janeiro (RJ): IBGE; 2016.

6. Instituto Nacional de Estatística (INE). Estatísticas demográficas 2015. Lisboa (PT): Instituto Nacional de Estatística; 2015.

7. Monteiro BR, Pisco AMSA, Candoso F, Bastos S, Reis M. Primary healthcare in Portugal: 10 years of contractualization of health services in the region of Lisbon. Ciência \& Saúde Coletiva, 2017;22 (3):725-36. http://dx.doi.org/10.1590/1413-81232017223.33462016

8. Escoval A, Santos ATL, Barbosa P. Contribution to the understanding of the health financingin Portugal: The case of primary healthcare, hospitals and long-term care. Tempus, actas de saúde colet. 2016;10(1): 17-27. https://doi.org/10.18569/tempus.v10i1.1858

9. Araújo GBF, de Miranda LO, Nolêto IRSG, de Aguiar WJL, Moreira AM, de Freitas DRJ. Comparation between the brazilian health system and the portuguese health system: general analysis. Sanare. 2017;16 (2):14-21. https://doi.org/10.20396/bjos.v16i0.8651056

10. Monteiro BR, Candoso F, Bastos S, Reis M. Analysis of the performance of contractualization primary health care indicators in the period 2009-2015 in Lisbon and Tagus Valley. Ciência \& Saúde coletiva. 2017;22(3):807-18. http://dx.doi.org/10.1590/1413-81232017223.33242016

11. Manso MEG, Maresti LTP, Oliveira HSB de. Analysis of quality of life and associated factors in a group of elderly persons with supplemental health plans in the city of São Paulo, Brazil. Rev. bras. geriatr. gerontol. 2019;22(4):e190013. https://doi.org/10.1590/1981-22562019022.190013.

12. World Health Organization (WHO). WHOQOL-Bref: introduction, administration, scoring and generic version of the assessment.Geneva: WHO,1996.

http: //www.who.int/mental_health/media/en/76.pdf

13. Polit DF. Fundamentos de pesquisa em enfermagem: avaliação de evidências para a prática da enfermagem. 7ed. Porto Alegre: Artmed, 2011.

14. Instituto Brasileiro de Geografia e Estatística (IBGE). Censo Demográfico censo sinopse 2010. Rio de Janeiro (RJ): IBGE; 2010. http://www.sidra.ibge.gov.br 
15. Sistema Nacional de Saúde (SNE). Relatório anual sobre o acesso a cuidados de saúde nos estabelecimentos de SNS e entidades convencionadas. Portugal (PT). SNE; 2015. https://www.sns.gov.pt/wp-content/uploads/2016/11/Relatório-Anual-sobre-o-Acesso-aCuidados-Saúde-no-SNS-2015-MS.pdf

16. Maleta CHM. Epidemiologia e Saúde pública. Belo Horizonte: COOPMED;2017.

17. Fleck MPA, Louzada S, Xavier M, Chachamovich E, Vieira G, Santos L, Pinzon V. Aplicação da versão em português do instrumento abreviado de avaliação de qualidade de vida WHOQOL-Bref. Rev. Saúde Pública. 2000;34(2):178-83.

https://doi.org/10.1590/S0034-89102000000200012

18.Serra A, Canavarro M, Simões M, Pereira M, Gameiro F, Quartilho M. Estudos psicométricos do instrumento de avaliação da Qualidade de Vida da Organização Mundial de Saúde (WHOQOL-Bref) para português de Portugal. Psiquiatria Clínica. 2006;27(1):31-40.

19. Bombardelli C, da Rosa LHT, Keller KD, Klahr PS, da Rosa PV, Peres A. Quality of life of elderly people living in a municipality with rural characteristics in the countryside of Rio Grande do Sul, Brazil. Rev. Bras. Geriatr. Gerontol. 2017;20(1):85-90.

https://doi.org/10.1590/1981-22562017020.160082

20. Barbosa KTF, Costa KNFM, Pontes MLF, Batista PSS, Oliveira FMRL de, Fernandes MGM. Envelhecimento e vulnerabilidade individual: um panorama dos idosos vinculados à estratégia saúde da família. Texto contexto \& enferm. 2017;26(2):e2700015.

http://dx.doi.org/10.1590/0104-07072017002700015

21. Castañeda-Flores T, Guerrero-Castañeda RF. Espiritualidad en adultos mayores hospitalizados: Aproximación cuantitativa. Rev Cuid. 2019;10(3):e724.

http://dx.doi.org/10.15649/cuidarte.v10i3.724

22. Rodrigues RMC, Azeredo ZAS, Mendes IMMMD, Crespo SSS, Silva CFR da. Os muito idosos do concelho de Coimbra: avaliação da funcionalidade na área de saúde física. Rev port saúde pública. 2016;34(2):163-72. http://dx.doi.org/10.1016/j.rpsp.2016.05.002

23. Nespollo AM, Marcon SR, Lima NVP, Dias TL, Martínez Espinosa M. Health Conditions and Memory Performance: a study with older adult women. Rev Bras Enferm. 2017; 70(3):6406. http://dx.doi.org/10.1590/0034-7167-2016-0529

24. Miranda LCV, Soares SM, Silva PAB. Quality of life and associated factors in elderly people at a Reference Center. Ciência \& Saúde Coletiva, 2016;21(11):3533-44.

http://dx.doi.org/10.1590/1413-812320152111.21352015

25. Garbaccio JL, Tonaco LAB, Estêvão WG, Barcelos BJ. Aging and quality of life of elderly people in rural areas. Rev Bras Enferm 2018;71(suppl 2):724-32.

http://dx.doi.org/10.1590/0034-7167-2017-0149

26. Leite MT, Castioni D, Kirchner RM, Hildebrandt LM. Capacidad funcional y nivel cognitivo de adultos mayores residentes en una comunidade em el sur de Brasil. Enferm. Glob. 2015;14(37):1-11. https://doi.org/10.6018/eglobal.14.1.196461

27. Guedes MBOG, Lima KC, Caldas CP, Veras RP. Social support and integral care to the health of the elderly. Physis. 2017;27(4):1185-1204.

http://dx.doi.org/10.1590/S0103-73312017000400017

28. Almeida-Brasil CC, Silveira MR, Silva KR, Lima MG, Faria CDCM, Cardoso CL, Menzel HJK, Ceccato MGB. Quality of life and associated characteristics: application of WHOQOLBREF in the context of Primary Health Care. Ciência \& Saúde Coletiva. 2017;22(5):1705-1716. http://dx.doi.org/10.1590/1413-81232017225.20362015

29. Gross CB, Kolankiewicz AC, Schmidt CR, Berlezi EM. Níveis de fragilidade de idosos e sua associação com as características sociodemográficas. Acta paul Enferm. 2018;31(2):209-16. http://dx.doi.org/10.1590/1982- 0194201800030 
30. Santos RMF, Santos PMF, Santos LDB. Duarte JC. Quality of life in older people: the case of Cova da Beira. Revista de Enfermagem Referência. 2013;III(11):37-48.

http://dx.doi.org/10.12707/RIII1210

31. Scolari GAS, Rissardo LK, Baldissera VDA, Carreira L. Emergency care units and dimensions of accessibility to health care for the elderly. Rev Bras Enferm. 2018;71(suppl 2):811-17. http://dx.doi.org/10.1590/0034-7167-2017-0440

32. Duarte A, Natércia J, Lapa F, Nunes C. Qualidade de vida e suporte social dos utentes da rede cuidados domiciliários. Psic., Saúde \& Doenças. 2014;15(3):623-34.

http://dx.doi.org/10.15309/14psd150306 